\title{
Chasing the ghost particle: The long and winding road toward the detection of solar neutrinos
}

\author{
Matteo Leone ${ }^{\text {a) }}$ \\ Department of Philosophy and Educational Sciences, University of Turin, 10124 Turin, Italy; Savigliano \\ Campus, University of Turin, 12038 Savigliano (CN), Italy; National Institute for Nuclear Physics (INFN), \\ Section of Turin; and Centro Fermi, 00184 Rome, Italy \\ Nadia Robotti ${ }^{\text {b) }}$ \\ Department of Physics, University of Genova, 16146 Genova, Italy; National Institute for Nuclear Physics \\ (INFN), Section of Genova; and Centro Fermi, 00184 Rome, Italy
}

(Received 6 November 2014; accepted 15 July 2015)

\begin{abstract}
One of the great achievements of neutrino physics was the discovery of solar neutrinos in 1968 through the Homestake underground experiment. This experiment exploited a radiochemical method based on the chlorine-argon process of inverse beta decay suggested by Bruno Pontecorvo in 1946 during his work in the classified Canadian nuclear project. In this paper, we study the emergence of the method. We focus on the role played by the problematic status of the neutrino and its antiparticle in its field of application and the influence exerted by the contemporary models of energy production in the sun. We also provide evidence that a first germ of this radiochemical method, in the form of a chlorine-sulfur process, was suggested in a paper published by Richard Crane in late 1930s. (c) 2015 American Association of Physics Teachers.
\end{abstract}

[http://dx.doi.org/10.1119/1.4927486]

\section{INTRODUCTION}

Since Wolfgang Pauli first postulated the neutrino in 1930 until the early 1950s, physicists generally considered it a sort of undetectable "ghost."1 During the 1933 Solvay Conference, James Chadwick (1935 Nobel Prize in Physics), who had recently discovered the neutron, said that "it is certain that the neutrino, if it exists, would be excessively hard to detect."2 Still in 1949, Luis Alvarez (1968 Nobel Prize) observed that "it is unfortunate that at the present time there is no convincing experimental proof that neutrinos exist."3

In this paper, we will address, mainly upon the basis of primary archival sources, the emergence in the 1930s of a radiochemical method for detecting neutrinos at a time when it was considered quite unrealistic to detect this particle. As it will be shown, almost 30 years later this method led to the discovery of solar neutrinos by Raymond Davis (2002 Nobel Prize).

\section{NEUTRINOS IN THE LATE 1930s AND EARLY 1940s: CONSENSUS AND EXPERIMENTAL EVIDENCE}

During the 1930s and 1940s, the most fruitful experimental method of investigating the existence of neutrino was the recoil method, where the energy and momentum of the recoil nucleus and of all other particles emitted in a beta disintegration decay process $\left(\mathrm{e}^{-}\right.$or $\left.\mathrm{e}^{+}\right)$are measured. Some of the most significant recoil experiments were performed in 1936 by Leipunski, ${ }^{4}$ in $1937-1939$ by Crane and Halpern, ${ }^{5}$ and in 1942 by Allen. ${ }^{6}$

In general, by the recoil experiments, it was found that energy and momentum are not conserved unless the neutrino is introduced to carry away the missing momentum and energy. Unfortunately, this method is a rather indirect one because the recoil experiments are interpreted on the basis of the assumption that the law of the conservation of energy and momentum hold in a single $\beta$ process. ${ }^{7}$

Direct evidence could be obtained only through experiments in which some characteristic process produced by neutrinos after they have been emitted is observed. The first type of experiments belonging to this class were those on the absorption of neutrinos through the measurements of their ionization effect, which were carried out in 1934-1935 at the Cavendish Laboratory in Cambridge. Of course, the expected chances of success were very low, since it was well known that "the neutrino would be very penetrating and would produce a very small specific ionization." 8 Thus, it came as no surprise when, in 1935 , by a pioneering underground neutrino experiment with a coincidence circuit of counters placed inside the Holborn Tube Station in London, Nahmias found that such a method was not suitable for detecting purposes. ${ }^{9}$

Actually, another unambiguous method to settle the matter of neutrino existence, besides looking at ionization effects, was known to exist soon after the formulation of Fermi's theory of beta decay. As outlined by Bethe and Peierls on February 20, 1934, such a method involves a reaction where "a neutrino hits a nucleus and a positive or negative electron is created while the neutrino disappears and the charge of the nucleus changes by $1, " 10$ i.e.,

$$
\nu+{ }_{Z}^{A} X \rightarrow e^{ \pm}+{ }_{Z \mp 1}^{A} X .
$$

Although this particular kind of "inverse $\beta$-process" is one process that a neutrino can certainly cause, it was later concluded that it is extremely rare. ${ }^{11}$ According to Bethe and Peierls, the cross-section for such a process may indeed be estimated "from the lifetime $t$ of $\beta$-radiating nuclei giving neutrinos of the same energy." This cross section resulted to have an upper threshold "of $10^{-44} \mathrm{~cm}^{2}$, corresponding to a penetrating power of $10^{16} \mathrm{~km}$ in solid matter," and "it is therefore absolutely impossible to observe processes of this kind with the neutrinos created in nuclear transformations."10 
Still, in his 1947 nuclear physics textbook, Bethe explained that this $10^{-44}$ order of magnitude- "given by the cross section for striking the nucleus (about $10^{-24} \mathrm{~cm}^{2}$ ) and the probability of $\beta$-decay within a nucleus (about $10^{-20} \mathrm{~cm}^{2}$ )" meant that the neutrino was "completely unobservable."12

As will be shown in the Sec. III, however, these negative expectations did not deter others from planning to detect the neutrino by the inverse $\beta$-process by late 1930 s and from being confident that this absolute impossibility might be successfully challenged in the near future.

\section{LOOKING FOR NEUTRINOS OUT OF A BAG OF SALT}

Notwithstanding the negative expectations, in 1939, after having attempted to detect the neutrino by the recoil method, University of Michigan physicist Richard Crane directed his attention toward the detection of the neutrino by a direct method. Since it was conclusively demonstrated that the presence of neutrinos cannot be detected by an ionization effect, ${ }^{9}$ Crane observed that "at least one possibility of detecting them remains, and that is by a process which is the reverse of the K-electron capture process." 13 This was just the process first proposed by Bethe and Peierls in 1934. ${ }^{10}$

According to Crane, ${ }^{13}$ if an inverse beta process was considered, chlorine-35 could be an adequate target; the expected reaction is

$$
C l^{35}+\nu \rightarrow S^{35}+e^{+} .
$$

As Crane observed, "the product ${ }^{35} \mathrm{~S}$ is a radioactive isotope [...] and decays back to ${ }^{35} \mathrm{Cl}$ with the emission of a negative electron and a neutrino" 13 according to the reaction

$$
S^{35} \rightarrow C l^{35}+e^{-}+\nu
$$

The existence of a neutrino could, therefore, be judged from the formation of the radioactive isotope ${ }^{35} \mathrm{~S}$. As later recollected by Crane, one of the reasons behind the choice of the ${ }^{35} \mathrm{Cl}^{35} \mathrm{~S}$ reaction was the small energy threshold. ${ }^{14}$ According to Crane, the minimum energy of a neutrino that can transform ${ }^{35} \mathrm{Cl}$ into ${ }^{35} \mathrm{~S}$ was $1.3 \mathrm{MeV}$, "a value well within the range of energies of neutrinos emitted from mesothorium $\left[{ }^{228} \mathrm{Ra}\right]$ and its products." ${ }^{13}$ Thus, mesothorium is suitable to be an optimal source of neutrinos in an attempt to induce a ${ }^{35} \mathrm{Cl}^{35} \mathrm{~S}$ reaction. The other, very important reason behind the choice of the ${ }^{35} \mathrm{Cl}^{35} \mathrm{~S}$ reaction was the ease of isolating the isotope possibly produced in the reaction induced by the neutrino, the ${ }^{35} \mathrm{~S}$ (by oxidizing the sulfur and later precipitating it as a sulfate).

These reasons made Crane confident about the possibilities of performing an experiment of this kind. In order to do this, he placed a capsule containing one $\mathrm{mCi}$ of mesothorium and its products in the center of a bag containing three pounds of $\mathrm{NaCl}$. After 90 days of irradiation, he extracted the sulfur and measured its possible activity in an ionization chamber. Unsurprisingly, no measurable beta activity was found in the sulfur. By drawing upon the estimate that $3 \times 10^{7}$ neutrinos of energy greater than $1.3 \mathrm{MeV}$ (the threshold energy for the transformation) are emitted per second by the source used, he concluded indeed that the sensitivity of the experiment was such that only a cross section as high as
$10^{-30} \mathrm{~cm}^{2}$ could have been detected, a far greater cross section than that expected by Bethe and Peierls $\left(10^{-44} \mathrm{~cm}^{2}\right)$.

Crane, however, was mainly interested in solar neutrinos. After having observed, on the basis of the recently developed Bethe theory of energy production in stars, that "a star generates about six percent of its energy in the form of neutrinos," 13 he took the view that it is of interest to know whether the neutrinos escape from the center of the sun without further collision with matter. He suggested, therefore, that if his bag of salt experiment was repeated again with a stronger source of mesothorium or radium, it could be possible to determine whether an absorption mechanism like the inverse beta process was great enough to prevent the escape of neutrinos from the sun. ${ }^{17}$

Even if he was interested in the flux of solar neutrinos, Crane did not discuss his ${ }^{35} \mathrm{Cl}^{-}{ }^{35} \mathrm{~S}$ method as a possible way to detect solar neutrinos. Yet, he implicitly referred to Bethe's theory of energy production in stars, which was based on the assumption that this energy is entirely due to the conversion of $\mathrm{H}$ to $\mathrm{He}$ and held that this conversion could occur through two different neutrino producing chains: the proton-proton $(p-p)$ chain, where ${ }^{2} \mathrm{H}$ and ${ }^{3} \mathrm{He}$ act as a catalyst that is created and destroyed during the intermediate steps of the fusion reaction, and the carbon-nitrogen-oxygen (CNO) cycle, where the $\mathrm{H} \rightarrow \mathrm{He}$ conversion is obtained by the action of these three elements as a catalyst. ${ }^{15}$

It was only in his January 1948 review paper about the state-of-art in the search for the neutrino that Crane hinted at the possibility of detecting solar neutrinos by this method. There, he stated that "the energy spectrum of the neutrinos produced in the [CNO] Bethe cycle is not unfavorable for the inverse beta process." 14 This possibility was actively pursued only several years later, though not by Crane, who gave up the method.

Significantly, in this same review paper, Crane emphasized also that "the use of the large neutrino flux from a chain-reacting pile to test for the inverse beta decay process has been a subject of conversation among physicists since the advent of the pile." He concluded that "it would be surprising if experiments of this sort were not going forward at the present time in one or more of the government laboratories." 14

Crane's guess was correct. Since 1945, another Italian nuclear physicist, who was working in a Canadian government laboratory as part of a classified project, had been thinking about detecting pile neutrinos through a method almost identical to that used by Crane, though with negative results, a few years earlier. His name was Bruno Pontecorvo.

\section{NEUTRINOS OUT OF A DRY CLEANING MACHINE}

By early 1943, and until 1949, Pontecorvo participated in a secret government project in Canada (Montreal and Chalk River) code-named Tube Alloys, consisting mainly of working on the design of a heavy water natural uranium reactor. During that period in Canada, he started thinking about the problem of neutrino detection. On May 19, 1945, he finished a note titled, "On a method for detecting free neutrinos,"16 (Fig. 1) that was issued two days later by the National Research Council of Canada (NRC) with the PD-141 code. ${ }^{17}$

For some reason, Pontecorvo never referred to this note in his scientific ${ }^{18}$ and autobiographical ${ }^{19}$ articles. The first reference was briefly made only in 1996 by Giuseppe 


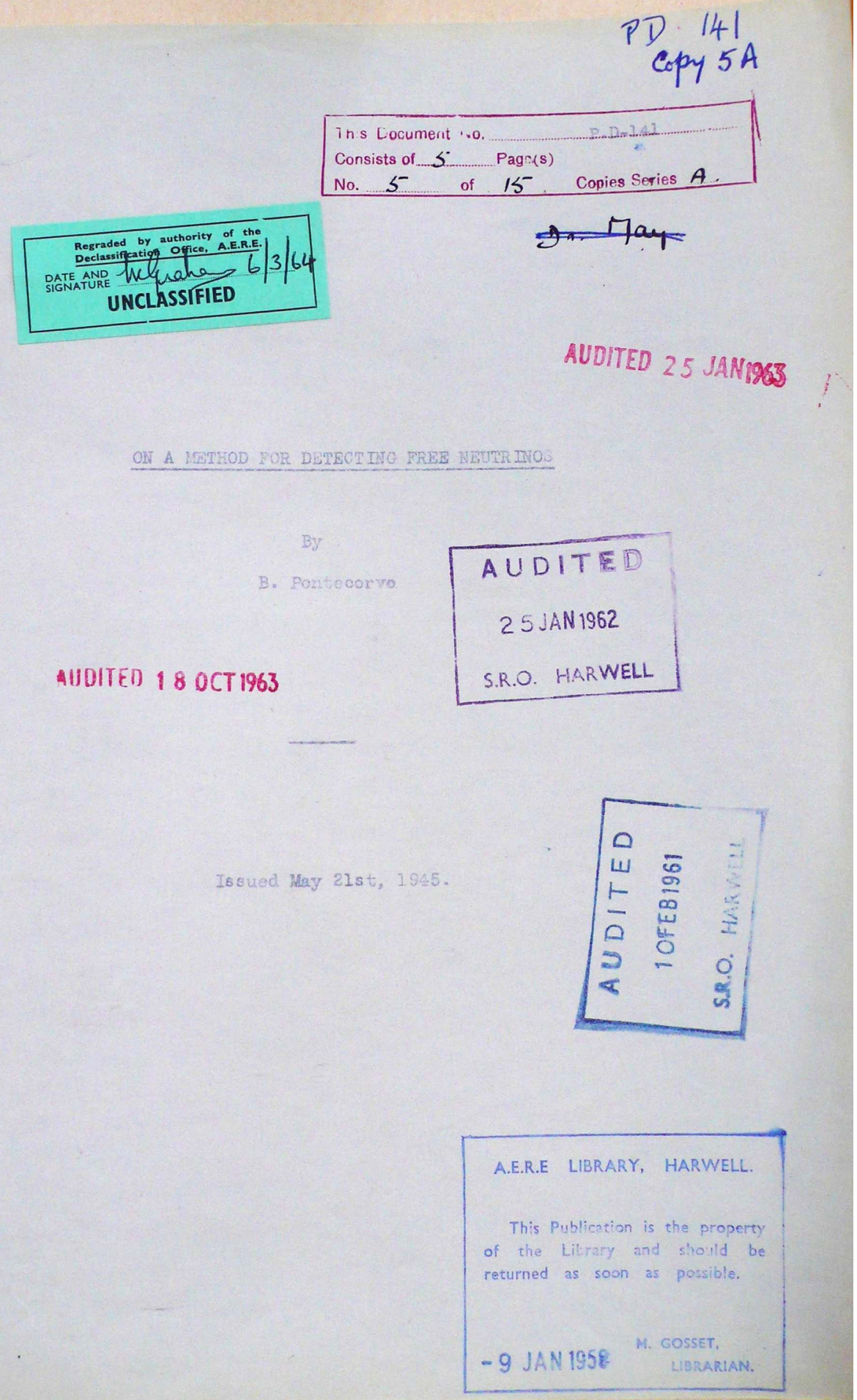

Fig. 1. Cover of the PD-141 report "On a method for detecting free neutrinos" (Courtesy: The National Archives of the UK, Ref. AB2/675).

Fidecaro, who got a copy of the report from Geoffrey Hanna, former collaborator of Pontecorvo in Canada. ${ }^{20}$

The object of Pontecorvo's note is to show that "the experimental observation of an inverse $\beta$ process is not out of the question," and to suggest "a method which might make an experimental observation feasible." ${ }^{\prime 16}$ Pontecorvo writes that even if the actual emission of the positive or negative electron when the neutrino is absorbed is not detectable in practice, "the radioactivity of the produced nucleus may be looked for and studied as an indication of the inverse $\beta$ process produced by neutrinos."
The essential point of this method is that the radioactive atoms produced have different chemical properties from the irradiated atoms. He suggests, therefore, that "it is possible (by means of the usual carrier technique) to extract from an irradiated volume of the order of cubic meters the radioactive atoms of known life-time," $" 16$ provided that some conditions are met. First, the material to be irradiated must be cheap, since cubic meters are involved. Second, the nucleus produced from the neutrino absorption must be radioactive with a lifetime of at least several days because of the long time involved in the chemical separation. Third, the 
chemical separation of the radioactive atoms must be simple. Finally, the masses of the original and final nuclei must have close values since the closer the masses, the larger the energy of the electron emitted concurrently with the absorbed neutrino and the larger the cross-section for an inverse $\beta$-ray process as a consequence of Fermi's $\beta$-ray theory. ${ }^{16}$

Given the above conditions, Pontecorvo re-discovered what Crane had already suggested, that "chlorine... fulfills reasonably well," in the form of ${ }^{35} \mathrm{Cl}$, and that ${ }^{35} \mathrm{~S}$ would be produced by absorption of a neutrino and emission of $\beta^{+}$via

$$
\nu+{ }_{17}^{35} \mathrm{Cl} \rightarrow \beta^{+}+{ }_{16}^{35} \mathrm{~S} \text { and }{ }_{16}^{35} \mathrm{~S} \stackrel{\beta^{-}}{\longrightarrow}{ }_{17}^{35} \mathrm{Cl} \text {. }
$$

Actually, Pontecorvo did not mention Crane's pioneering attempt to detect the neutrino by the inverse beta process. What is remarkable is that Pontecorvo instead cites the other contemporary Crane and Halpern papers about the recoil method in the same journal. It is unclear whether the Italian physicist arrived independently to suggest a chlorine-sulfur radiochemical method or if he was consciously influenced by Crane's paper but for some reason decided to not cite it.

Differently from Crane, who had suggested using $\mathrm{NaCl}$, Pontecorvo identifies carbon tetrachloride $\left(\mathrm{CCl}_{4}\right)$, a wellknown and cheap dry cleaning solvent at the time, as the best chlorine compound to irradiate from a chemical point of view. ${ }^{21}$ Upon the basis of the 1944 Seaborg's Table of Isotopes, ${ }^{22}$ where ${ }^{35} \mathrm{~S}$ is listed as beta-decaying to ${ }^{35} \mathrm{Cl}$ with a period of 87.1 days (the energy of the $\beta$-ray radiation being only $120 \mathrm{keV}$ ), Pontecorvo argues for a much larger scale experiment than that previously considered by Crane, stating

[The] production of ${ }^{35} \mathrm{~S}$ would be observable by using a volume of $\mathrm{CCl}_{4}$ of the order of cubic meters and radioactive source having intensity of the order of $10^{17}$ neutrinos/s. Such extremely intense source does not go much beyond the present technical facilities ("hot" metal from pile). ${ }^{16}$

Maurice Pryce, member of Tube Alloys, had pointed out to Pontecorvo that the sun is another possible source since it emits a "quite considerable" flux of neutrinos. ${ }^{23}$ To Pontecorvo, this source, however, does not seem appropriate to the radiochemical method because "the flux of neutrinos received from the sun $\left[10^{10}\right.$ neutrinos $\mathrm{cm}^{-2} \mathrm{~s}^{-1}$ providing Bethe's carbon cycle is the source of energy of the sun] is too low for an experiment of the type suggested." 16

The contents of PD-141 show that Pontecorvo's optimism as to the possibility of experimentally observing an inverse- $\beta$ process by the radiochemical method was rooted in the new possibilities offered by nuclear reactors, such as the heavy water nuclear reactor soon to be available in Chalk River. If the natural sources usually available before the advent of fission reactors were measured in terms of activities of the order of one curie or $3.7 \times 10^{10}$ disintegrations/s (corresponding to $3.7 \times 10^{10}$ neutrinos/s or a neutrino flux at one meter of about $3 \times 10^{5} \mathrm{~cm}^{-2} \mathrm{~s}^{-1}$ ), the fission reactors were expected to yield a $10^{7}-10^{8}$ higher neutrino flux. As a matter of comparison, the original 2-kW Fermi pile produced $4 \times 10^{14}$ neutrinos/s. While this by itself was no guarantee that the neutrino detection problem was solvable, it made Pontecorvo's bet worth pursuing, provided that the technical difficulties inherent in the observations of a few atoms of a radioisotope out of a massive volume of dry cleaning machine liquid were successfully overcome.
One year after the drafting of PD-141, Pontecorvo presented a lecture titled "Inverse Beta Process" to a nuclear physics conference held in Montreal. ${ }^{24}$ On November 20, 1946, the contents of this lecture were issued again as an NRC report with the PD-205 code (Fig. 2). ${ }^{25}$

PD-205 was originally classified by the U.S. AEC because of its sensitive content concerning reactor physics. In fact, Pontecorvo's report was declassified less than two years later, on October 8, 1948 (a few days before two other Montreal conference NRC reports on different topics). ${ }^{26}$ Thus, Pontecorvo's second report soon became available to the scientific community, quite contrary to the first one that, for unclear reasons, was declassified only in 1964 though it had the same sensitive contents.

The analysis of the contents of the second Pontecorvo report on neutrino detection reveals strong similarities with the earlier note. In this second report, we indeed find again the bold conjecture that the experimental observation of the inverse $\beta$ process "is not out of the question," and the conditions to be fulfilled to make this observation feasible. Further, the conclusion that chlorine would fulfill them reasonably well, in the form $\mathrm{CCl}_{4}$, and the estimate that a volume of the order of $1 \mathrm{~m}^{3}$ of $\mathrm{CCl}_{4}$ would require a neutrino source that, while extremely intense, "is not too far from what could be obtained with present day facilities." 25

However, in the previous report, Pontecorvo suggested, as Crane had earlier, a reaction starting from ${ }^{35} \mathrm{Cl}$, which accounts for about $76 \%$ of the natural chlorine. In his second report, he focuses instead on ${ }^{37} \mathrm{Cl}$ ( $24 \%$ of natural chlorine). The reaction is now

$$
\nu+{ }_{17}^{35} \mathrm{Cl} \rightarrow \beta^{-}+{ }_{18}^{37} \mathrm{Ar} \quad \text { and } \quad{ }_{18}^{37} \mathrm{Ar} \stackrel{\mathrm{Ke}}{\longrightarrow}{ }_{17}^{35} \mathrm{Cl}
$$

where ${ }^{37} \mathrm{Ar}$ decays into ${ }^{37} \mathrm{Cl}$ by $\mathrm{K}$ capture with a half-life of 34 days.

Pontecorvo's new attention for ${ }^{37} \mathrm{Cl}$ as opposed to the previous ${ }^{35} \mathrm{Cl}$ is a matter of speculation because no reference to PD-141 is made in his second report and in his later publications. What is clear is that at some point he became aware that relatively fewer difficulties in the extraction of argon might be expected if compared with the separation of sulfur out of chlorine. When only chemical methods are possible, as is the case for separating ${ }^{35} \mathrm{~S}$, "it is necessary that the addition of only a few grams of a non-isotopic carrier, per hundred liters of material treated gives an efficient separation." 25 It is, therefore, a difficult separation. On the contrary, if the nucleus formed in the inverse $\beta$ process is a rare gas, as in the case of argon out of chlorine, the separation is easier since it can be obtained by physical methods, "for example by boiling the material irradiated." ${ }^{25}$ As emphasized by Pontecorvo, Otto Frisch, who was former head of the Manhattan Project's Critical Assembly Group and who had spent a period in Chalk River in March $1946,{ }^{27}$ agreed with the Italian physicist that the chemical separation obtained by physical methods was "the most promising method." 25

A further element behind Pontecorvo's new choice might be discovering at some point between the two reports the favorable decay mode of ${ }^{37} \mathrm{Ar}$ by $\mathrm{K}$ electron capture. ${ }^{28}$ This discovery may have supported the view that $\mathrm{Cl}-\mathrm{Ar}$ was the optimal method for detecting the neutrino.

Pontecorvo also reaffirms that the solar neutrino flux is several orders of magnitude lower than necessary to obtain an observable effect and that the only available strong 


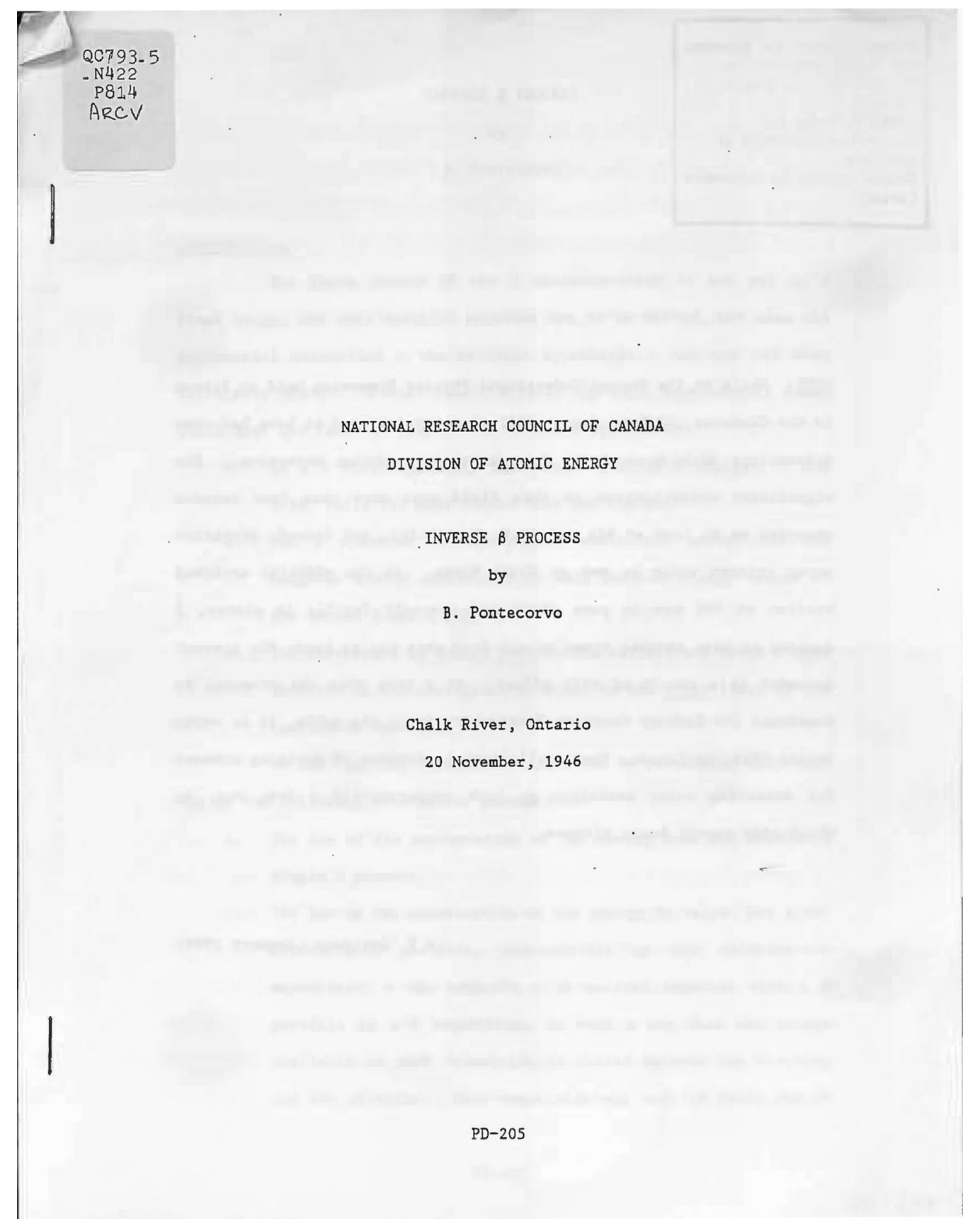

Fig. 2. Cover of the PD-205 report "Inverse $\beta$ Process" (Courtesy: National Research Council Canada Archives).

neutrino sources are related to nuclear reactors: either the "hot" uranium metal extracted from a pile or, still better, "the pile itself, during operation." 25 The chlorine-argon method indeed required a neutrino flux of the order of $10^{14}$ neutrinos $\mathrm{s}^{-1} \mathrm{~cm}^{-2}$ that, "though extremely high, is not too far from what could be obtained with present day facilities." 25

In April 1949, a few months after the declassification of PD-205, Alvarez discussed in a detailed fashion Pontecorvo's Cl-Ar reaction in a University of California report, giving widespread visibility to the method. Alvarez supported Pontecorvo's conclusions on both reactor and solar neutrinos by quantitatively showing the feasibility of the method for detecting reactor neutrinos and by briefly commenting on the negligibility of the expected effects of solar neutrinos. 24
In an interesting turn of events, experiments in the next two decades clearly showed that the opposite was true and that only solar neutrinos could be captured by Pontecorvo's net.

\section{THE SOLAR NEUTRINOS, AT LAST}

In February 1949, Pontecorvo moved to England to join the Harwell nuclear facility, where he planned "to do the experiments with neutrinos from a pile," though it was "a highly acrobatic feat." 29 In fact, Pontecorvo never conducted the planned neutrino experiment. In the late summer of 1950, he defected to the Soviet Union, ${ }^{30}$ where he "happened to work in an accelerator laboratory (and not in a reactor laboratory)" "19 and, therefore, stopped working on the neutrino detection problem. 
In the meantime, a new theoretical point was raised and brought to Pontecorvo's attention, though without no significant effect on his research program. In July 1948, following a visit to Chalk River and recent conversations with Fermi, Anthony Turkevich, a University of Chicago physicist, wrote to Pontecorvo that:

[The] theoreticians have not yet decided [if] there is a "real" difference between a "neutrino" and an "anti-neutrino." In this case I wonder if the pile ( $\beta^{-}$decays) which furnishes "anti-neutrinos" can induce a process such as $\mathrm{Cl}^{37}\left(\nu, \mathrm{e}^{-}\right) \mathrm{A}^{37}$ which really requires "neutrinos.",

At that time, it was indeed unknown whether the neutrino $\nu_{-}$emitted in the $\beta^{-}$decay (named antineutrino) via

$$
n \rightarrow p+\beta^{-}+\nu_{-}
$$

and the neutrino $\nu_{+}$emitted in the $\beta^{+}$decay (the neutrino) via

$$
p \rightarrow n+\beta^{+}+\nu_{+}
$$

were identical or if they differed. In particular, it was unknown if the neutrino in the equivalent inverse- $\beta$ process (8), corresponding to process (6) of

$$
\nu_{+}+n \rightarrow p+\beta^{-}
$$

could be considered as the same particle as the one in the inverse- $\beta$ process (9), corresponding to process (7) of

$$
\nu_{-}+p \rightarrow n+\beta^{+} \text {. }
$$

This situation dramatically changed only in the 1950s when awareness was gained that neutrinos (produced in fusion reactions, as in the sun) and antineutrinos (produced by reactors and radioactive sources) are not identical. This was achieved through experimental confirmation that reaction (9) actually occurs if reactor antineutrinos are used and experimental (dis)confirmation that reaction (8) does not take place if the same reactor antineutrinos are employed.

In particular, in 1956, Frederick Reines and Clyde Cowan, who for several years had been focusing on the inverse process (9) in Hanford and at Savannah River, succeeded to detect reactor antineutrinos $\nu_{-}$through a method completely different from the radiochemical one, the recently emerged technique of organic liquid scintillators. ${ }^{32}$ The intense flux of antineutrinos produced in the beta decay of fission fragments enters the scintillator containing a high proportion of hydrogen and, therefore, of protons. By reaction (9), the antineutrino interacts with one of these protons, creating a positron and a neutron that are revealed as a pair of delayed signals. In 1995, about 20 years after the death of Cowan, Reines was awarded the Nobel Prize in Physics "for the detection of the neutrino" (actually, an antineutrino).

With hindsight, we can look back and conclude that the first, conceptually correct attempt to detect antineutrinos $\nu_{-}$through inverse process (9) was just Crane's 1939 chlorinesulfur reaction. This possible road for detecting the antineutrino, however, was never pursued again because it was, in the meantime, surpassed by Reines and Cowan's method.

As regards inverse process (8), it was first taken in serious consideration by Pontecorvo in his second report, where he suggested using the ${ }^{37} \mathrm{Cl}^{37} \mathrm{Ar}$ reaction (5) for detecting reactor neutrinos. This experiment was first conducted by Raymond Davis, Jr., in 1954 under the hypothesis that if $\nu_{+}$and $\nu_{-}$do not differ, "one should be able to observe the process upon carrying the experiment to the required sensitivity." ${ }^{33}$ Davis exposed up to $11,400 \mathrm{l}$ of $\mathrm{CCl}_{4}$ to $\nu_{-}$emitted by the Brookhaven reactor and later by the Savannah River reactor without finding evidence of a reaction induced by reactor antineutrinos, even if the required sensitivity was obtained. ${ }^{34}$

The positive result obtained by Reines and Cowan with reaction (9), coupled with the negative result obtained by Davis with reaction (8), both using reactor antineutrinos, supported the view that though "in the theory of beta decay it is not a priori evident that neutrinos and antineutrinos are distinguishable," these "two independent studies of the inverse beta reaction have established with good probability that such is the case." 35

Therefore, this outcome stimulated Davis to produce Pontecorvo's ${ }^{37} \mathrm{Cl}^{37} \mathrm{Ar}$ reaction by solar $\nu_{+}$neutrinos. This choice required, however, the capability of observing much lower fluxes of energetic neutrinos with respect to those available near a large reactor ${ }^{36}$ and a new theoretical framework on solar neutrinos. Both conditions were met only in the mid-1960s, when the development of models about energy production in the sun offered encouraging prospects for solar neutrino detection.

By the time of Bethe's theory, it was indeed understood that a natural laboratory for producing an important flux of $\left(\nu_{+}\right)$neutrinos was the sun. In the mid-1950s, however, it was unclear whether solar neutrinos were energetic enough to overcome the threshold of the chlorine-argon reaction. While in the previous decade Pontecorvo's ${ }^{37} \mathrm{Cl}^{37} \mathrm{Ar}$ method could in principle be used to detect solar neutrinos, since Bethe's CNO nuclear cycle (which was believed to be the most important source of energy in the sun) predicted the production of neutrinos whose maximum energy $(1.8 \mathrm{MeV})^{15}$ was known to be larger than the threshold of neutrino energy for transforming ${ }^{37} \mathrm{Cl}$ into ${ }^{37} \mathrm{Ar}$ $(0.816 \mathrm{MeV}),{ }^{37}$ in the $1950 \mathrm{~s}$, such an expectation was quite less legitimate.

In the early $1950 \mathrm{~s}$, it was indeed found that in the sun the $\mathrm{p}-\mathrm{p}$ chain-where the reaction of two protons leads to the successive production of the deuteron, ${ }^{3} \mathrm{He}$, and ${ }^{4} \mathrm{He}-$ outweighs the $\mathrm{CNO}$ cycle by a considerable factor under the assumed conditions of temperature and density. ${ }^{38}$ Since the neutrinos emitted in the $\mathrm{p}$-p chain were known to have a maximum energy of $0.41 \mathrm{MeV}$ (Ref. 39) (below the $0.816 \mathrm{MeV}$ threshold), a chlorine-argon experiment to detect neutrinos from the sun did not seem feasible.

Things changed in 1958 with Holmgren and Johnston's discovery that the probability was 2500 times higher than previously estimated ${ }^{40}$ that the $p$-p chain led to a branch with production of ${ }^{7} \mathrm{Be}$ out of ${ }^{3} \mathrm{He}$ and a ${ }^{4} \mathrm{He}$ nuclei. As was pointed out by William Fowler, the successive formation of ${ }^{8} \mathrm{~B}$ by proton capture on ${ }^{7} \mathrm{Be}$ resulted in the emission of a substantial flux of energetic neutrinos of up to $14.1 \mathrm{MeV}$ in energy that "may be detectable through observations on ${ }^{37} \mathrm{Cl}\left(\nu, \beta^{-}\right)^{37} \mathrm{Ar}$, using the technique developed by Davis" with reactor neutrinos. ${ }^{41}$

Contrary to Fowler's expectations, in 1960 , the ${ }^{7} \mathrm{Be} \rightarrow{ }^{8} \mathrm{~B}$ proton capture reaction rate was found to be too disappointingly low to ensure a negative result even with a very large volume of chlorine. ${ }^{42}$ The expectations of a chlorine-argon experiment seemed brighter only by 1964 when John Bahcall theoretically found that the capture rate of ${ }^{8} \mathrm{~B}$ 
neutrinos by chlorine was significantly higher than expected. The cross-section of Pontecorvo's reaction (5) was indeed found by Bahcall to be so large for ${ }^{8} \mathrm{~B}$ energetic neutrinos that they were expected to produce about $90 \%$ of the total signal in a detector based upon the chlorine-argon reaction. ${ }^{43}$

The above encouraging estimates set the stage for the Davis' well-known solar neutrino experiment. At first, a pilot experiment using 1,000 gal of perchloroetylene $\left(\mathrm{C}_{2} \mathrm{Cl}_{4}\right)$ buried in a limestone mine in Ohio $\left(\mathrm{C}_{2} \mathrm{Cl}_{4}\right.$ was chosen instead of $\mathrm{CCl}_{4}$ because its vapor pressure was lower and it was eight times less toxic) ${ }^{44}$ enabled setting limits on the extraterrestrial neutrino flux. Then, in 1965-1966, a large-scale experiment using a 100,000 gal detector was set up in the Homestake Gold Mine in South Dakota (Fig. 3). The Homestake experiment apparatus was located $1,480 \mathrm{~m}$ underground to reduce the production of ${ }^{37} \mathrm{Ar}$ from $(\mathrm{p}, \mathrm{n})$ reactions by protons formed in cosmic-ray muon interactions. At that depth, the expected ${ }^{37} \mathrm{Ar}$ production from all background processes was estimated to be less than 0.2 atoms/day, which was well below the rate expected from solar neutrinos (3.5 atoms/day). ${ }^{45}$

The Homestake experiment was a success, since Pontecorvo's method worked and solar neutrinos were found. However, it was a problematic success because there were not as many as were expected. In 1968, the first results of the experiment led to an upper limit of the solar neutrino flux corresponding to a capture rate of $0.5 /$ days, ${ }^{45}$ much lower than the theoretical estimate (that, in the meantime, new calculations had lowered to a value two to three times higher). ${ }^{46}$ This result was confirmed by the measurements in the 1970-1994 period, when Davis' experiment had been running almost continuously. An estimated total of 2,200 argon atoms were produced in the tank, i.e., "the sun produces one third as many neutrinos as expected."47 This gap between measurements and calculations of solar neutrino flux has since been known as the "solar neutrino problem."

With Davis' experiment, a "new field of neutrino physics" was born, ${ }^{48}$ a field to which Pontecorvo continued to give significant contributions. One notable example was his idea, put forward in 1969, that the solar neutrino problem might be solved through "neutrino oscillations" that would cause a decrease in the number of detectable solar neutrinos at the earth's surface. ${ }^{49}$

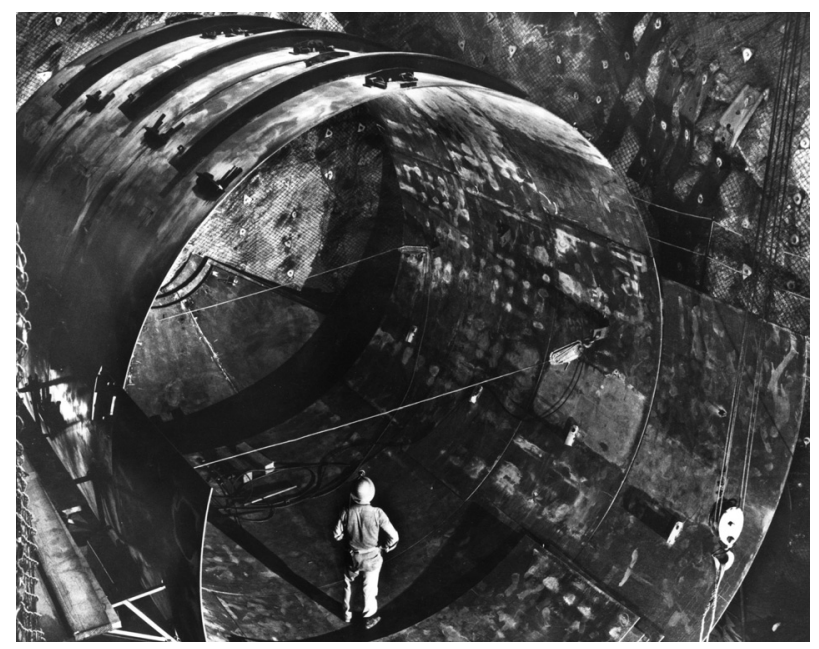

Fig. 3. Construction of the tank used in the solar neutrino experiment in the Homestake gold mine (Courtesy: Brookhaven National Laboratory).
Davis' results were confirmed in the late 1980s by Masatoshi Koshiba and his group with another gigantic detector built in Japan, called Kamiokande, based on the completely different water Cherenkov technique, where solar neutrinos could be detected by the elastic scattering of neutrinos on electrons. These accomplishments earned Davis and Koshiba one half of the 2002 Nobel Prize in Physics. Significantly, in his Nobel lecture, Davis openly acknowledged his debt to Pontecorvo when he wrote that the method behind the radiochemical experiment "was suggested by Pontecorvo."47

\section{CONCLUDING REMARKS}

The present account shows that the radiochemical method used by Davis had a long history dating back to the times when it was felt possible to get direct evidence of the neutrino by an inverse beta decay experiment actually performed by Crane, using a $\mathrm{Cl}-\mathrm{S}$ reaction, a little bag of table salt as a sample, and a capsule of radionuclide as a source. Crane's presently forgotten experiment was later, being unaware or not, proposed again by Pontecorvo in his equally forgotten first NRC report, where, through a large scale experiment, a considerable volume of chlorine-based dry-cleaning liquid was expected to enjoy success in detecting the neutrinos produced by the emerging technology of nuclear reactors. A better method to detect pile neutrinos was eventually devised by Pontecorvo, using a different reaction scheme (Cl-Ar) involving the same dry-cleaning liquid but a different chlorine isotope, with the advantage of a much easier chemical separation.

This method played a relevant twofold role in the hands of Davis. When applied to study reactor neutrinos, the method brought to light the different behavior of the neutrino and antineutrino, thus paving the way for the detection of a different kind of neutrino, those emitted by sun. Second, in 1968, it proved successful in detecting solar neutrinos.

The emergence of solar neutrinos out of the Homestake experiment was a formidable accomplishment requiring the successful mastering of the extraction of a few atoms out of $10^{30}$. However, the 30 -year-long historical process that made this possible was shaped by the evolving models of energy production in stars that, in turn, made a radiochemical experiment to detect neutrinos from the sun more-or-less feasible. At the end of this long and winding road, when all the conditions proved to be favorable for the experiment thought of many years before by Pontecorvo, the experiment was finally performed, leading to the detection of solar neutrinos for the first time ever.

\section{ACKNOWLEDGMENTS}

Part of this work was presented as invited lectures at the 2013 and 2014 National Conferences of the Italian Physical Society. This work benefited greatly from access to documents preserved in the National Archives (London), the Amaldi Archives (Sapienza University of Rome), and the Churchill Archives Centre (Cambridge), retrieved within the context of a project leading to the International symposium "The legacy of Bruno Pontecorvo: the man and the scientist" (Rome, September 2013). The authors are also grateful to S. Leclair, NRC Archival Service Officer, for a copy of PD205; to W. F. Davidson, NRC, for information on PD-205; and to F. Guerra, Sapienza University of Rome, for useful 
discussions and helpful advice. This work was supported with institutional research grants from the University of Turin and the University of Genova. The authors are also grateful to INFN and Centro Fermi for financial support.

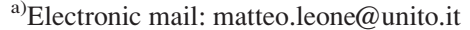

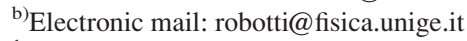

${ }^{1}$ F. Reines, "The neutrino: From poltergeist to particle," Rev. Mod. Phys. 68, 317-327 (1996). On the early history of neutrino, see F. Guerra, M. Leone, and N. Robotti, "When energy conservation seems to fail: The prediction of the neutrino," Sci. Educ. 23, 1339-1359 (2014).

${ }^{2}$ J. Chadwick, "Discussion du rapport de M. Heisenberg," in Structures et propriétés des noyaux atomiques, Proceedings of the VII Solvay Conference, Brussels, 22-29 October (Gauthier-Villars, Paris, 1934), pp. 325-326.

${ }^{3}$ L. Alvarez, "A proposed experimental test of the neutrino theory," Report No. UCRL-328 (University of California, Berkeley, 1949).

${ }^{4}$ A. I. Leipunski, "Determination of the energy distribution of recoil atoms during $\beta$ decay and the existence of the neutrino," Math. Proc. Cambridge 32, 301-303 (1936).

${ }^{5}$ H. R. Crane and J. Halpern, "New experimental evidence for the existence of a neutrino," Phys. Rev. 53, 789-794 (1938); "Further experiments on the recoil of the nucleus in beta-decay," 56, 232-237 (1939). On 22 May 1938, they also got The New York Times coverage with the article "Cloud-chamber test finds neutrino real."

${ }^{6} \mathrm{~J} . \mathrm{S}$. Allen, "Experimental evidence for the existence of a neutrino," Phys. Rev. 61, 692-697 (1942).

${ }^{7}$ B. Pontecorvo, "The neutrino and the recoil of nuclei in beta disintegrations," Rep. Prog. Phys. 11, 32-42 (1947).

${ }^{8}$ J. Chadwick and D. E. Lea, "An attempt to detect a neutral particle of small mass," Math. Proc. Cambridge 30, 59-61 (1934).

${ }^{9}$ M. E. Nahmias, "An attempt to detect the neutrino," Math. Proc. Cambridge 31, 99-107 (1935).

${ }^{10}$ H. Bethe and R. Peierls, "The "neutrino'," Nature 133, 532 (1934).

${ }^{11}$ H. Bethe and R. F. Bacher, "Nuclear physics. A. Stationary states of nuclei," Rev. Mod. Phys. 8, 82-229 (1936).

${ }^{12}$ H. Bethe, Elementary Nuclear Theory (Wiley \& Sons, New York, 1947).

${ }^{13}$ H. R. Crane, "An attempt to observe the absorption of neutrinos," Phys. Rev. 55, 501-502 (1939).

${ }^{14} \mathrm{H}$. R. Crane, "The energy and momentum relations in the beta-decay and the search for the neutrino," Rev. Mod. Phys. 20, 278-295 (1948).

${ }^{15}$ H. A. Bethe, "Energy production in stars," Phys. Rev. 55, 434-456 (1939).

${ }^{16} \mathrm{~B}$. Pontecorvo, "On a method for detecting free neutrinos," National Research Council of Canada Report No. PD 141 (21 May 1945), AB 2/ 675, The National Archives, Kew Gardens, London (TNA).

${ }^{17}$ F. E. Stratton, Report number series used by the Technical Information Service in cataloguing reports, TID-85, 2nd revised ed. (U.S. AEC, Oak Ridge, Tennessee, 1960).

${ }^{18} \mathrm{~B}$. Pontecorvo selected scientific works, recollections on B. Pontecorvo, edited by S. M. Bilenky et al. (SIF, Bologna, 1997); 2nd ed. (SIF, Bologna, 2013).

${ }^{19}$ B. Pontecorvo, "The infancy and youth of neutrino physics: Some recollections," J. Phys. 43, C8-221-C8-236 (1982); "Pages in the development of neutrino physics," Sov. Phys. Usp. 26, 1087-1108 (1983).

${ }^{20}$ G. Fidecaro, "Bruno Pontecorvo: From Rome to Dubna," in Ref. 24, p. 21-29. See also G. Fidecaro, "Bruno Pontecorvo, and his inverse $\beta$ process to detect neutrinos: A page of history," in The Legacy of Bruno Pontecorvo: The Man and The Scientist, edited by L. Bonolis, C. Dionisi, and N. Robotti, Conference Proceedings, Rome, 11-12 September 2013 (SIF, Bologna, 2014), pp. 137-152.

${ }^{21}$ This was suggested to Pontecorvo by Jules Gueron, a French physical chemist member of Tube Alloys.

${ }^{22}$ G. T. Seaborg, "Table of isotopes," Rev. Mod. Phys. 16, 1-32 (1944).

${ }^{23}$ Pontecorvo thanks Pryce "for very useful discussion and advice" (see Ref. 9, p. 4). Pryce was well known for a paper on the idea that the photon is a composite particle formed of a neutrino-antineutrino pair, which he proved wrong.

${ }^{24}$ Program of Nuclear Physics Conference, GBR/0014/PNVO 4-1-3. The papers of Bruno Pontecorvo. Churchill Archives Centre, Churchill College, Cambridge (CAC). In summer, he had considered giving a communication to a Los Alamos Conference before discovering that the Atomic Energy Act "does not permit aliens to enter activities of the Manhattan District" (N. E. Bradbury, letters to B. Pontecorvo (11 July and 9 August 1946), GBR/0014/PNVO 4.1.1. CAC.

${ }^{25}$ B. Pontecorvo, "Inverse $\beta$ process," National Research Council of Canada (NRC) Report No. PD 205 (20 November 1946), QC793-5-N422 P814 ARCV.

${ }^{26}$ United States Government Publications, Monthly Catalog issued by the Superintendent of Documents, Nos. 648-659 (U.S. Government Printing Office, Washington, January-December 1949).

${ }^{27}$ O. R. Frisch, letters to B. Pontecorvo (4 and 21 February 1946), GBR/ 0014/PNVO 4.1.1. CAC.

${ }^{28}$ P. K. Weimer, J. D. Kurbatov, and M. L. Pool, "K-electron capture in radioactive argon A 37 ," Phys. Rev. 66, 209-214 (1944). While this paper was published a few months before PD-141, it appeared in print after the publication of Seaborg's table used by Pontecorvo as source about decay modes and half lives.

${ }^{29}$ B. Pontecorvo, letter to E. Amaldi (19 June 1949). Box 142, Amaldi Archives, Sapienza University of Rome.

${ }^{30}$ Pontecorvo worked until his death in what is now known as the Joint Institute for Nuclear Research in Dubna.

${ }^{31}$ A. L. Turkevich, letter to B. Pontecorvo (26 July 1948), GBR/0014/PNVO 4.1.1. CAC.

${ }^{32}$ C. L. Cowan et al., "Detection of the free neutrino: A confirmation," Science 124, 103-104 (1956).

${ }^{33} \mathrm{R}$. Davis, Jr., "Attempt to detect the antineutrinos from a nuclear reactor by the $\mathrm{Cl}^{37}\left(\bar{v}, e^{-}\right) \mathrm{A}^{37}$ reaction," Phys. Rev. 97, 766-769 (1955).

${ }^{34} \mathrm{R}$. Davis, Jr., "An attempt to detect the neutrinos from a nuclear reactor by the $\mathrm{Cl}^{37}\left(\bar{v}, e^{-}\right) \mathrm{A}^{37}$ reaction," Bull. Am. Phys. Soc. 1, 219 (1956).

${ }^{35}$ C. O. Muehlhause and S. Oleksa, "Antineutrino flux from a reactor," Phys. Rev. 105, 1332-1334 (1957).

${ }^{36} \mathrm{R}$. Davis, Jr. and D. S. Harmer, "Solar neutrino detection by the $\mathrm{Cl}^{37}-\mathrm{Ar}^{37}$ method," Informal Conference on Experimental Neutrino Physics (CERN, Geneva, 20-22 January 1965), pp. 201-212.

${ }^{37}$ This experimental value was obtained by the measurement of the ${ }^{37} \mathrm{Ar}-{ }^{37} \mathrm{Cl}$ mass difference (H. T. Richards, R. V. Smith, and C. P. Browne, "Proton-neutron reactions and thresholds," Phys. Rev. 80, 524-530 (1950)). See also Ref. 14.

${ }^{38}$ E. A. Frieman and L. Motz, "The proton-proton reaction and energy production in the sun," Phys. Rev. 89, 648-650 (1953).

${ }^{39}$ E. E. Salpeter, "Nuclear reactions in the stars. I. Proton-proton chain," Phys. Rev. 88, 547-553 (1952).

${ }^{40}$ H. D. Holmgren and R. L. Johnston, " $\mathrm{He}^{3}(\alpha, \gamma) \mathrm{Be}^{7}$ reaction," Bull. Am. Phys. Soc. 3, 26 (1958).

${ }^{41}$ W. A. Fowler, "Completion of the proton-proton reaction chain and the possibility of energetic neutrino emission by hot stars," Astrophys. J. 127, 551-556 (1958).

${ }^{42} 2.5 \times 10^{7}$ neutrinos $/ \mathrm{cm}^{2} \mathrm{~s}$ at earth from the proton capture reaction as opposed to $1.2 \times 10^{10}$ neutrinos $/ \mathrm{cm}^{2} \mathrm{~s}$ from the electron capture reaction (R. W. Kavanagh, "Proton capture in $\mathrm{Be}^{7}$," Nucl. Phys. 15, 411-420 (1960)).

${ }^{43}$ J. N. Bahcall, "Solar neutrino cross sections and nuclear beta decay," Phys. Rev. 135, B137-B146 (1964).

${ }^{44}$ R. Davis, Jr., B. T. Cleveland, and J. K. Rowley, "Underground science at Homestake," Workshop on Science Underground, Los Alamos, 26 September-1 October 1982.

${ }^{45}$ R. Davis, Jr., D. S. Harmer, and K. C. Hoffman, "A search for neutrinos from the Sun,” Phys. Rev. Lett. 20, 1205-1209 (1968).

${ }^{46}$ J. N. Bahcall, N. A. Bahcall, and G. Shaviv, "Present status of the theoretical predictions for the ${ }^{37} \mathrm{Cl}$ solar-neutrino experiment," Phys. Rev. Lett. 20, 1209-1212 (1968).

${ }^{47}$ R. Davis, "Nobel lecture: A half century with solar neutrinos," Rev. Mod. Phys. 75, 985-994 (2003).

${ }^{48}$ The Royal Swedish Academy of Science, 8 October 2002, Neutrino astronomy, Advanced information on the Nobel Prize in Physics 2002. $<$ http://www.nobelprize.org/nobel_prizes/physics/laureates/2002/ advanced-physicsprize2002.pdf $>$

${ }^{49}$ V. Gribov and B. Pontecorvo, "Neutrino astronomy and lepton charge," Phys. Lett. B 28, 493-496 (1969). 